\title{
The ENUBET project: high precision neutrino flux measurements in conventional neutrino beams
}

\section{F. Terranova*}

Phys. Dep., Univ. di Milano Bicocca and INFN Sezione di Milano Bicocca

E-mail: francesco.terranova@cern.ch

G. Ballerini ${ }^{\mathrm{a}, \mathrm{b}}$ A. Berra ${ }^{\mathrm{a}, \mathrm{b}}$ R. Boanta ${ }^{\mathrm{b}, \mathrm{h}}$ M. Bonesini ${ }^{\mathrm{b}}$, C. Brizzolari ${ }^{\mathrm{a}, \mathrm{b}}$, M. Calviani $^{\mathrm{m}}$, M.G. Catanesi ${ }^{1}$, S. Cecchini ${ }^{\mathrm{c}}$, F. Cindolo ${ }^{\mathrm{c}}$, A. Coffani $^{\mathrm{b}, \mathrm{h}}$, G. Collazuol ${ }^{\mathrm{k}, \mathrm{j}}$, E. Conti F. Dal Corso ${ }^{j}$, G. De Rosa ${ }^{\mathrm{p}, \mathrm{q}}$, A. Gola ${ }^{\mathrm{o}}$, R.A. Intonti ${ }^{1}$, C. Jollet ${ }^{\mathrm{d}, \mathrm{s}}$, Y. Kudenkor, M. Laveder ${ }^{\mathrm{k}, \mathrm{j}}$, A. Longhin ${ }^{\mathrm{j}}$, P.F. Loverre ${ }^{\mathrm{n}, \mathrm{f}}$, L. Ludovici ${ }^{\mathrm{f}}$, L. Magaletti ${ }^{\mathrm{l}}$, G. Mandriolic ${ }^{\mathrm{c}}$, A. Margotti ${ }^{\mathrm{c}}$, V. Mascagna ${ }^{\mathrm{a}, \mathrm{b}}$, N. Mauri ${ }^{\mathrm{c}}$, A. Meregaglia ${ }^{\mathrm{s}}$, M. Mezzetto $^{\mathrm{j}}$, M. Nessi $^{\mathrm{m}}$, A. Paoloni ${ }^{\mathrm{e}}$, M. Pari ${ }^{\mathrm{k} j}$, G. Paternoster ${ }^{\circ}$, L. Patriziic ${ }^{\mathrm{c}}$ C. Piemonte $^{\mathrm{o}}$, M. Pozzato ${ }^{\mathrm{c}}$, M. Prest ${ }^{\mathrm{a}, \mathrm{b}}$, F. Pupilli ${ }^{\mathrm{e}}$, E. Radicioni ${ }^{1}$, C. Riccio ${ }^{\mathrm{p}, \mathrm{q}}$, A. C. Ruggeri ${ }^{\mathrm{p}}$, M. Soldani ${ }^{\mathrm{a}, \mathrm{b}}$ G. Sirric ${ }^{\mathrm{c}}$ M. Tenti ${ }^{\mathrm{c}, \mathrm{g}}$ E. Vallazza ${ }^{\mathrm{i}}$, L. Votano ${ }^{\mathrm{e}}$, E. Wildner $^{\mathrm{m}}$

a Phys. Dep. Università degli Studi dell'Insubria, via Valeggio 11, Como, Italy

b INFN, Sezione di Milano-Bicocca, piazza della Scienza 3, Milano, Italy

${ }^{\mathrm{c}}$ INFN, Sezione di Bologna, viale Berti-Pichat 6/2, Bologna, Italy

d IPHC, Université de Strasbourg, CNRS/IN2P3, Strasbourg, France

e INFN, Laboratori Nazionali di Frascati, via Fermi 40, Frascati (Rome), Italy

${ }^{\mathrm{f}}$ INFN, Sezione di Roma 1, piazzale A. Moro 2, Rome, Italy

g Phys. Dep. Università di Bologna, viale Berti-Pichat 6/2, Bologna, Italy

h Phys. Dep. Università di Milano-Bicocca, piazza della Scienza 3, Milano, Italy

${ }^{\mathrm{i}}$ INFN Sezione di Trieste, via Valerio, 2 - Trieste, Italy

j INFN Sezione di Padova, via Marzolo, 8 - Padova, Italy

${ }^{k}$ Phys. Dep. Università di Padova, via Marzolo, 8 - Padova, Italy

${ }^{1}$ INFN Sezione di Bari, via Amendola, 173 - Bari, Italy

${ }^{\mathrm{m}}$ CERN, Geneva, Switzerland

${ }^{\mathrm{n}}$ Phys. Dep. Università La Sapienza, piazzale A. Moro 2, Rome, Italy.

${ }^{\circ}$ Fondazione Bruno Kessler (FBK) and INFN TIFPA, Trento, Italy

p INFN, Sezione di Napoli, via Cinthia, 80126, Napoli, Italy

q Phys. Dep. Università degli Studi di Napoli Federico II, via Cinthia, 80126, Napoli

${ }^{\mathrm{r}}$ Institute of Nuclear Research of the Russian Academy of Science, Moscow, Russia

${ }^{s}$ CENBG, Université de Bordeaux, CNRS/IN2P3, 33175 Gradignan, France

The ENUBET Collaboration is developing a technology to reduce by one order of magnitude the uncertainty on fluxes in conventional neutrino beam. The ENUBET beamline exploits the large angle production of positrons from $K^{+} \rightarrow e^{+} \pi^{0} v_{e}$ in the decay tunnel to monitor the associated production of $v_{e}$. This method provides the $v_{e}$ rate at source at the $1 \%$ level. In this talk, we will summarize the results during the first year of the project and plans up to completion (2021).

The European Physical Society Conference on High Energy Physics

5-12 July, 2017

Venice 


\section{ENUBET}

Flux uncertainties represent the main source of systematic errors in all neutrino cross section measurements. This uncertainty also affects the $v_{e}$ and $\bar{v}_{e}$ cross sections, which are poorly known in spite of their relevance for precision oscillation physics in the next generation of long-baseline experiments. Most of the sources of systematics are due to the full simulation of the beamline needed to estimate the flux. A direct monitoring of the leptons produced in the decay tunnel overcomes such limitation if the leptons can be measured with a precision significantly better than the usual muon monitoring performed after the beam dump. If the decay tunnel is sufficiently short $(50 \mathrm{~m})$ and the momentum of secondary hadrons amounts to a few $\mathrm{GeV}$, the only source of $v_{e}$ in the beamline is due to the three body decay of the charged kaons $\left(K_{e 3}\right): K^{+} \rightarrow e^{+} \pi^{0} v_{e}$. Due to the large mass of the kaon, the positrons are emitted at an average angle $(\sim 100 \mathrm{mrad})$ much higher than the muon emission angle from the $\pi^{+} \rightarrow \mu^{+} v_{\mu}$. As a consequence, the lateral walls of the decay tunnel can be instrumented by fast $(<10 \mathrm{~ns})$ calorimetric modules to separate positrons from other charged particles. Photons produced by $\pi^{0}$ decays are vetoed by plastic scintillator pads located just below the calorimeter ("photon veto"). The photon veto also provides a precise timing (1 ns) of the positron $\left(t_{0}\right)$. Beamlines equipped with such instrumentation are called "monitored neutrino beams" and were proposed in 2015 for the precise measurement of the electron neutrino cross section [1].

The ENUBET Project is aimed at demonstrating the technical feasibility and physics performance of monitored neutrino beams. The Project is funded by the European Research Council (ERC Consolidator Grant, PI A. Longhin, host institution INFN) from June 2016 to May 2021. More recently the Project has been awarded additional funds from the Italian ministry for education and science (MIUR) to develop the timing system below $1 \mathrm{~ns}$, which is of relevance for the "tagged neutrino beams" [2]. Since December 2016, ENUBET is framed in the CERN Neutrino Platform under the NP3-Plafond R\&D programme [2].

\section{Progress during the first year}

In 2017 the geometry of the tunnel and detector $\left(6 \times 6 \times 10 \mathrm{~m}^{3}\right)$ was re-optimized with respect to [1] and physics performances have been significantly improved. A 500 ton neutrino detector with a transverse size of $6 \times 6 \mathrm{~m}^{2}$ located $50 \mathrm{~m}$ after the beam dump of the ENUBET beamline will accumulate (see Fig. 1) $10^{4} v_{e}$ CC events $\left(1 \%\right.$ statistical uncertainty on $\sigma_{v_{e}}$ ) with $1.0 \times 10^{20}$ protons on target (pot) at the JPARC synchrotron $(30 \mathrm{GeV})$. The same result can be obtained at the Fermilab Main Ring $(120 \mathrm{GeV})$ with $2.4 \times 10^{19}$ pot and at the CERN SPS $(400 \mathrm{GeV})$ with $1.1 \times 10^{19}$ pot. It corresponds to $2-3$ months of data taking at current intensity for such proton drivers.

The Collaboration is developing the focusing system both assuming a conventional pulsed horn and a static system. The optimization of the focusing system and the design of the transfer line is ongoing. In particular, the optimization of the line (dipoles and quadrupoles) is performed with TRANSPORT. The implementation and full simulation of particle transport, collimation and re-interaction is carried out with G4Beamline. Assessment of the doses is addressed using FLUKA.

\footnotetext{
* Speaker.
} 
Event rates. $0.5 \mathrm{kt}, 1.0 \mathrm{e}+20$ pot, $\mathrm{L}=0.1 \mathrm{~km}$

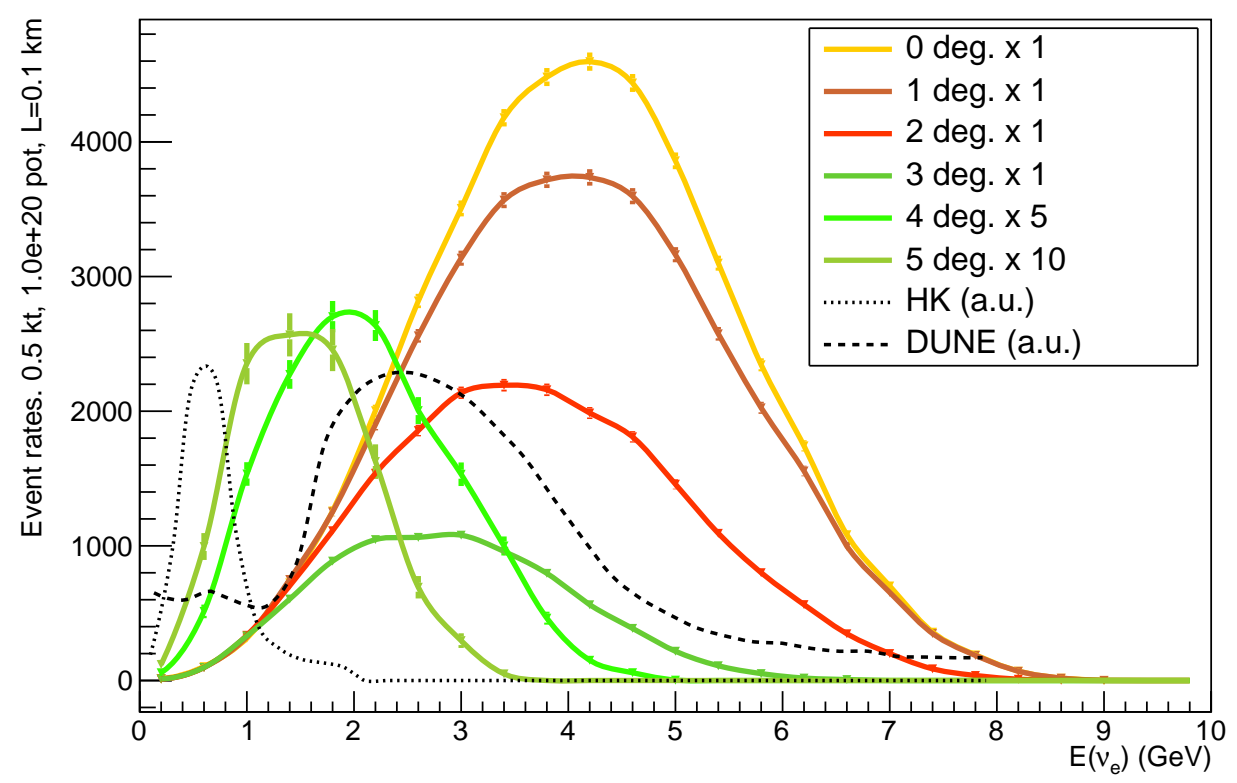

Figure 1: $v_{e} \mathrm{CC}$ events at the neutrino detector (500 tons) as a function of the neutrino energy. The beamline is optimized for the energy range of interest for DUNE. The rates are shown at different off-axis angles of the detector $\left(0^{\circ}-5^{\circ}\right)$. The events correspond to $1 \times 10^{20}$ pot at the Fermilab Main Ring $(120 \mathrm{GeV})$ and the detector is located $50 \mathrm{~m}$ after the beam dump $(0.1 \mathrm{~km}$ after the entrance of the decay tunnel). The dotted and dashed lines show the Hyper-Kamiokande and DUNE neutrino spectrum, respectively.

In parallel with the simulation of the transfer line, the GEANT4 simulation of the decay tunnel and instrumentation has been completed. Positron identification was estimated on the basis of the rate and particle distribution of Ref. [1] and will be updated once the study of the focusing and transfer line is completed. The algorithms for particle identification [3] employ the energy deposit pattern in the calorimeter modules to separate electromagnetic from hadronic showers. The information from the photon veto is exploited to cluster the modules belonging to the same event (event building) and separate photons from charged particles. Assuming the rates of [1] $\left(<500 \mathrm{kHz} / \mathrm{cm}^{2}\right)$, a full simulation of the pile-up is now available. The calorimetric modules are positioned at a $1 \mathrm{~m}$ radius from the axis of the decay tunnel and the ENUBET instrumentation provides positron identification with an efficiency of $25 \%$ and a signal-to-noise ratio of $\sim 1$. The statistical error on positron monitoring is negligible and the assessment of the systematics due to the detector response is ongoing.

Ionizing and non-ionizing doses at the instrumentation were evaluated with FLUKA 2011 and are shown in Fig. 2. Integrated doses corresponding to $10^{4} v_{e} \mathrm{CC}$ events at the neutrino detector amount to $<0.05 \mathrm{kGy}$ and $1.9 \times 10^{11} \mathrm{n} / \mathrm{cm}^{2}$ ( $1 \mathrm{MeV}$ equivalent). These estimates validate the possibility of using conventional plastic scintillators as an active medium for the calorimeter and photon veto.

The reference detector option for ENUBET is based on $3 \times 3 \times 10 \mathrm{~cm}^{3}$ ultra-compact modules (UCM) that sample the e.m. shower every $4.3 X_{0}$ [4, 5]. The UCM modules tested in 2016 and 2017 
are made of $1.5 \mathrm{~cm}$ thick iron slabs interleaved with $0.5 \mathrm{~cm}$ plastic scintillators. The scintillation light is collected by Kuraray Y11 wavelength shifter fibers (1 mm diameter) running through the UCM, i.e. through five iron+scintillator tiles and read out by SiPM. The fibers are inserted into a 3D printed plastic mask located downstream of each UCM. The grooves in the mask allow to couple the SiPMs that are soldered on a printed circuit board with the WLS fibers. This design has been tested at the CERN East Area facility with electrons, muons and pions up to $5 \mathrm{GeV}$, i.e. in the energy range of interest for ENUBET. The results of the test $[6,7,8]$ demonstrate that the design is appropriate for the needs of positron monitoring and validate the full simulation of the decay tunnel.

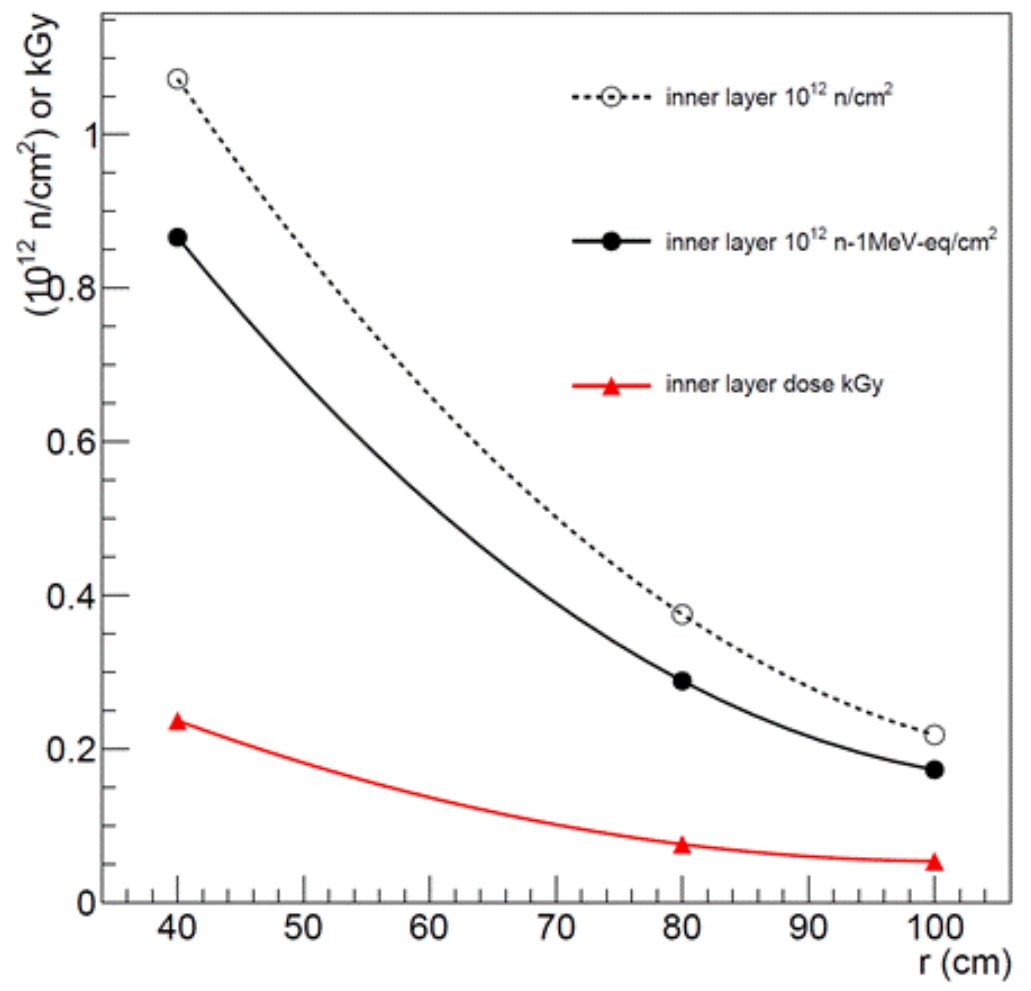

Figure 2: Ionizing dose (red line, in kGy), neutron fluence (black dashed line, in $\mathrm{n} / \mathrm{cm}^{2}$ ) and $1 \mathrm{MeV}$ equivalent neutron fluence (black continuous line) in the innermost modules of the calorimeter as a function of the tunnel radius.

The reference detector option requires SiPM embedded in the bulk of the calorimeters and, hence, exposed to radiation. Since SiPM are sensitive to neutron damage, a dedicated irradiation test has been performed at the CN irradiation facility at INFN-LNL (Legnaro) in summer 2017. SiPM of different pixel size produced by FBK were irradiated up to $10^{12} \mathrm{n} / \mathrm{cm}^{2}$ (1 MeV-eq.) and their response in the UCM were tested at the CERN East Area facility [8].

Finally, injection molded scintillators that are suitable for large scale production have been successfully produced by Uniplast (Russia) and tested at CERN in July 2017. Non conventional options based on polisiloxane scintillators to avoid drilling or injection molding are also under investigation. 


\section{Acknowledgment}

This project has received funding from the European Union's Horizon 2020 Research and Innovation programme under Grant Agreement no. 654168 and no. 681647.

\section{References}

[1] A. Longhin, L. Ludovici and F. Terranova, Eur. Phys. J. C 75 (2015) 155.

[2] A. Berra et al., "Enabling precise measurements of flux in accelerator neutrino beams: the ENUBET project” CERN-SPSC-2016-036; SPSC-EOI-014.

[3] A. Meregaglia et al., JINST 11 (2016) C12040.

[4] A. Berra et al., Nucl. Instrum. Meth. A 830 (2016) 345

[5] A. Berra et al., Nucl. Instrum. Meth. A 845 (2017) 511.

[6] A. Berra et al., IEEE Trans. Nucl. Sci. 64 (2017) 1056.

[7] V. Mascagna, Talk at the 8th International Conference on New Development in Photodetectors (NDIP 2017), Tours, France, 3-7 July 2017.

[8] A. Berra et al., in preparation. 